Review Article

\title{
The Response of an Oral Surgery Department during the COVID-19 Pandemic
}

Sandhu BK*, Blanchard J and Koshal S

Royal National ENT and Eastman Dental Hospitals, University College London Hospitals, London

\begin{abstract}
As we begin to see the light at the end of the tunnel and some routine activity beginning, we are slowly adapting to our new 'normal'. The question is when will true normality resume, and what the effect this momentary pause will have for the future of our profession.

At the Royal National ENT and Eastman Dental Hospitals, a new service was established during the COVID-19 pandemic, to provide urgent dental care to the communities of North, East and Central London. We use this article to share our experiences and approach of tackling the COVID-19 pandemic in a busy elective Oral Surgery department, where there was not an existing urgent dental care service. This article demonstrates the steps, processes and considerations taken in setting up an urgent dental hub in a short period of time to tackle the consequence of the global pandemic on dental care. We highlight the management strategy implemented including the barriers and challenges faced by an urgent dental care centre at a time when primary dental care was unavailable, highlighting the lessons learnt that can be used and further developed in the case of a further peak or pandemic in the future.
\end{abstract}

Keywords: Response; Oral surgery; Coronavirus; Pandemic

\section{Introduction}

As we start to see the light at the end of the tunnel, we begin to question how long will this appear as our new normal? We see changes in our routine Personal Protective Equipment (PPE), restrictions for our shielded patients and a much slower pace of our daily service.

Coronavirus Disease (COVID-19), stems from the virus severe acute respiratory Syndrome Coronavirus 2 (SARS-CoV-2), which contains an enveloped spherical particle with glycoprotein clubshaped projections [1] measuring up to 0.14 microns in size [2]. The Health Safety Executive recommends the use of Face Filtering Piece 3 (FFP3) respirators for use against viruses to control exposure to the lowest levels. However, they accept FFP2 level respirator protection if stocks are low during a pandemic [3]. This is consistent with guidance issued by the World Health Organisation, with focus on performing a 'seal check' at all times when using these respirators [4] which can filter $99 \%$ of airborne particles [5]. FFP3 respirators can be limited to Aerosol Generating Procedures (AGPs) alongside full facial shields or visors as these respirators are not fluid repellent, a long- sleeved fluid repellent gown (covering arms) and gloves [6]. The World Health Organisation defines an AGP as a procedure resulting in the production of airborne particles less than 5 micrometres in size, which can be transported across the air possibly resulting in infection if inhaled, and has established dental procedures including high speed drilling and ultrasonic scaling as high risk AGPs [7].

Citation: Sandhu BK, Blanchard J, Koshal S. The Response of an Oral Surgery Department during the COVID-19 Pandemic. Dentist. 2020; 2(1): 1015.

\section{Copyright: () 2020 Sandhu BK}

Publisher Name: Medtext Publications LLC

Manuscript compiled: Sep $04^{\text {th }}, 2020$

*Corresponding author: Baljinder Kaur Sandhu, Royal National ENT and Eastman Dental Hospitals, University College London Hospitals, 47-49 Huntley Street, London, E-mail: baljindersandhu@nhs.net
With the opening of multiple UDC (Urgent Dental Centre) service providers across England, protocols were put into place regarding social distancing and appropriate zoning measures with particular focus on patients with suspected/confirmed COVID-19, those shielded and those at higher risk [4].

We use this article to share our experiences and approach of tackling the COVID-19 pandemic in a busy elective Oral Surgery department, where there was not an existing urgent dental service. This article outlines the processes and considerations taken in rapidly setting up an urgent dental hub for the provision of care for patients in London (Figure 1).

\section{The Eastman Dental Hospital Response Immediate phase}

All elective dental care provided in the primary care setting was halted on $25^{\text {th }}$ March 2020, following a letter from the Chief Dental Officer sent to all practices in England [8]. This required many dental practices to provide a telephone triage service, with appropriate direction provided for patients in need of emergency treatment.

Commissioners issued NHS local regions to initiate the planning of local UDCs, and the use of 111 triage services. There was also encouragement for the production of appropriate local Safety Operating Procedure protocols.

Across the Eastman Dental Hospital, a UDC service provider was set up, offering face-to face consultations and treatment facilities for patients requiring emergency dental treatment. This arrangement was initiated following a remote risk assessment and telephone triage.

The UDC service was provided jointly with the Restorative Dentistry Department, allowing for an efficient and cohesive service with detailed assessment and restorative options for patients. The service was led each day by Restorative and Oral Surgery consultants working together to appropriately streamline patients (Figure 2).

Prior to setting up the UDC, an 'Oral Surgery Complications' guide was issued to all clinicians of the service. This guide included 


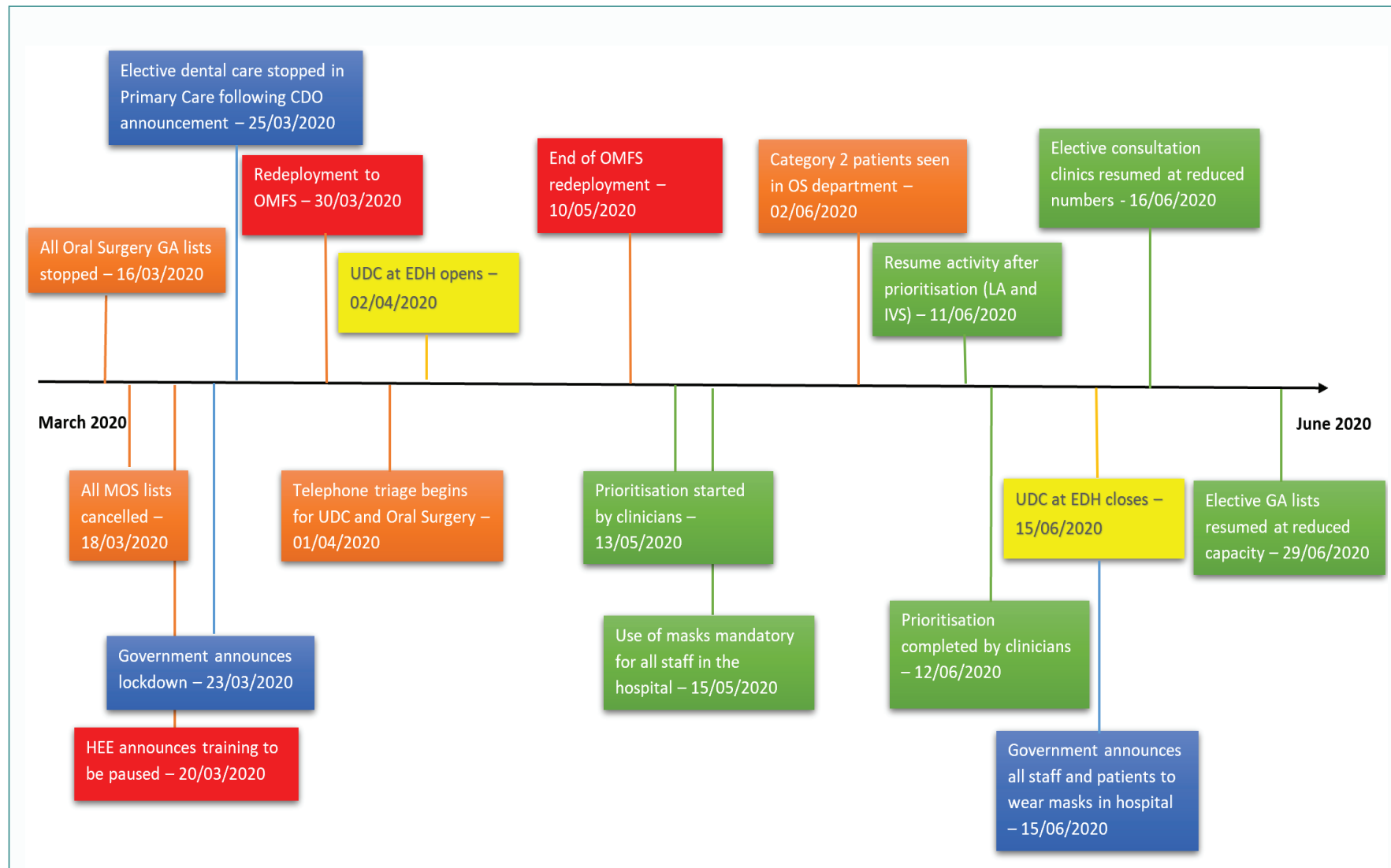

Figure 1: A timeline showing the sequential changes adapted to our service during the COVID-19 pandemic.

1. Pain not controlled with regular use of painkillers, tooth assessed and deemed to require extraction

2. Oro-facial swelling that is significant and worsening

3. Limited opening $<10 \mathrm{~mm}$ and associated pain and swelling

4. Oro-dental conditions that are likely to exacerbate systemic medical conditions

5. Post-extraction bleeding that the patient is not able to control with local measures

6. Dental trauma

7. Ulcers/suspicious lesions

8. Head and Neck Cancer patients pre-radiotherapy/ pre-surgery

Figure 2: The acceptance criteria of patients to be seen in the UDC service.

management of an oro-antral communication, tuberosity fracture and uncontrolled bleeding. In addition, as a department it was agreed that AGPs were to be limited where possible, particularly regarding apical root fracture during a non-surgical extraction. It was advised to leave $3 \mathrm{~mm}$ or less apices in situ, which had no associated pathology, if extensive bone removal was required for retrieval or during a lengthy and complex surgical extraction to reduce risk of prolonged exposure. Furthermore, each patient seen was triaged using systematic approach as outlined below (Figure 3).

Recent literature highlighted the link between intraoral radiography and the induction of cough reflexes. Bali et al. [9] suggests the use of extraoral imaging preferentially to intraoral image for this specific reason.

In addition to the provision of urgent dental care, the Oral Surgery Department were redeployed to provide assistance to the
Oral and Maxillofacial Surgery team, through joining the emergency on-call trauma rota due to medically qualified OMFS clinicians being redeployed to join other medical specialities. The extended roles of the dentally qualified Oral Surgery clinicians included the acute management of odontogenic facial infections and swellings, facial fractures and surgical closure of lacerations. This crossover service between both specialties also enabled a follow up service for patients in the Oral Surgery department, for example patients with facial swellings were able to be seen in the outpatient setting, freeing up space in the emergency department.

With the cessation of primary care services in England a significant number of dental paediatric patients with acute swellings presented to Accident and Emergency, resulting in emergency general anaesthetic procedures carried out at a separate site.

At the Eastman Dental Hospital, elective general anaesthetic procedures were suspended on $17^{\text {th }}$ March to allow the utilisation of theatre space for intensive care facility conversion as well as the anaesthetic department focus shifting to caring for COVID-19 patients.

The beginning of the pandemic also saw the pause to training programmes for clinicians, including the foundation dental programme, dental core training and specialty training. At the Eastman Dental Hospital, it led to the specific redeployment of dental core trainees to in-house testing, telephone triage and the maternity department.

\section{Provision of care}

The UDC service also enabled urgent dental care to be provided 


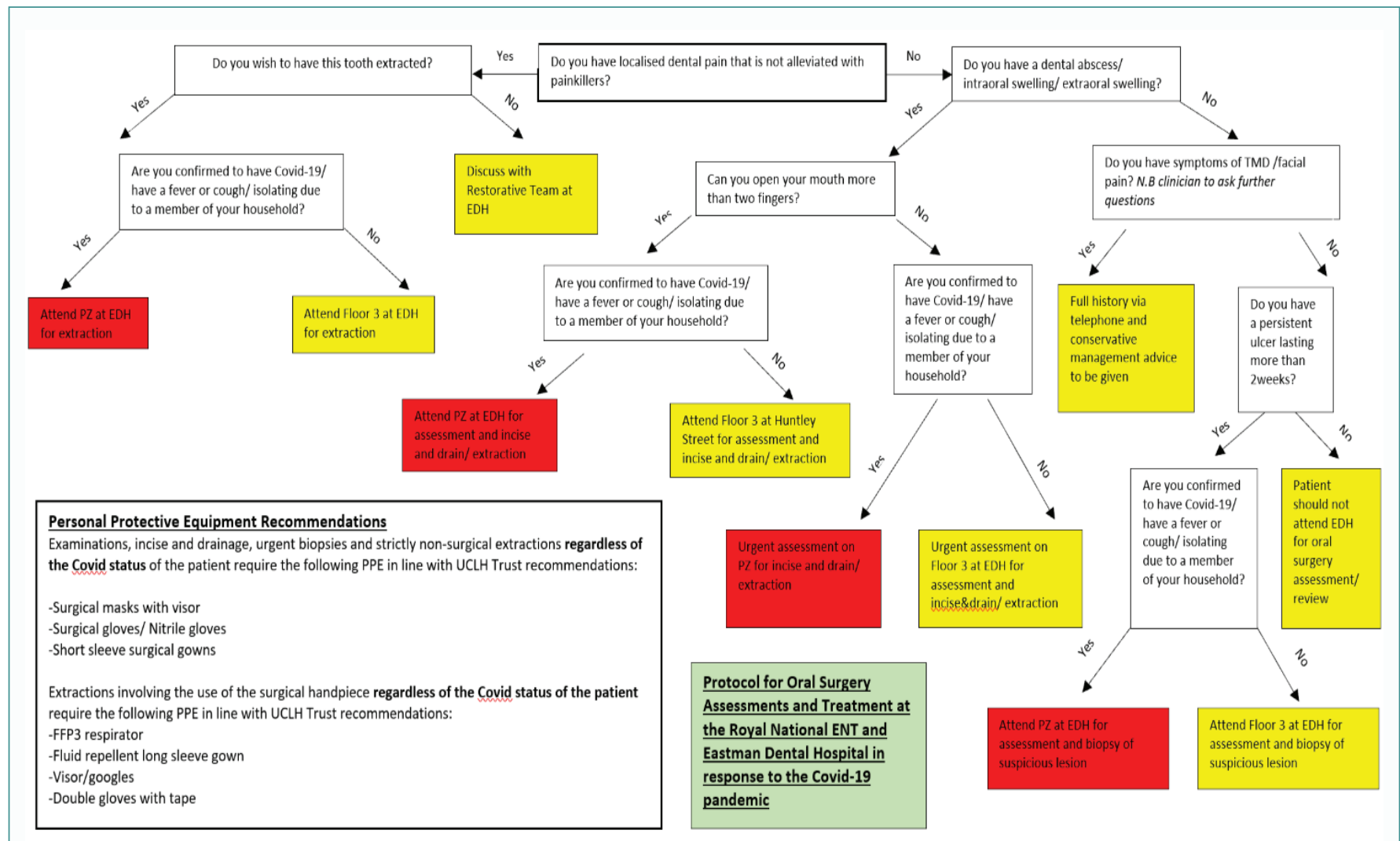

Figure 3: A flowchart highlighting the triaging process for an urgent presenting patient.

for shielded groups with appropriate separation of suspected or confirmed COVID-19 cases. This was carried out through appropriate zoning of the hospital, using a segregated floor particularly for suspected/confirmed COVID-19 cases during the height of the pandemic with peak number of cases, and later the provision of care at dedicated times during the clinic day. Every patient entering the hospital was asked to use alcohol sanitising gel and on arrival to the UDC floor, alongside the use of a surgical fluid repellent mask. All patients who were to be treated on the suspected/confirmed floor, were asked to use an allocated lift to the clinic and also provided with a surgical mask as recommended by Public Health England [10]. The hospital had existing specific patient and staff zones, which aided the limitation of spread and contamination through separation of patients and staff on entering and exiting the building (Figure 4).

In addition, further zoning was carried out within the UDC level of the hospital, through labelling of doors as 'AGP' and 'Non-AGP' zones. In particular, all AGPs were carried out in rooms with closed doors with treatment undertaken by clinicians who were deemed suitably trained and confident to complete the procedure.

Government recommendations of social distancing were observed throughout the hospital with markers placed on the floors, one- way system flow for patients and clinicians including restrictions in common areas. All members of staff were advised to wear face masks at all times from the $15^{\text {th }}$ May.

Desktop computer access was also limited in neighbouring positions with an increase in the use of computers of wheels. Remote access working was encouraging whereby feasible to undertake clinical tasks.
Following an aerosol generating procedure, 20 minutes of time was allowed in each of these rooms for adequate time for clearance of aerosols. This is the recommended time in an isolation room with 1012 air changes per hour [10]. It has been stated that AGPs should only take place in rooms with a minimum of 6 air changes per hour, and all listed rooms for AGPs have between 13.8-16.9 changes per hour.

\section{Recovery phase}

The fallow period of 10 minutes was implemented in the returning to service phase with elective patient treatment starting from the $11^{\text {th }}$ June 2020 , with a $50 \%$ reduction in patient number for all minor oral surgery under LA and IV sedation clinics, allowing adequate time for the settling of aerosols between patients. This change was made as a result of a new standard operative procedure at the Trust.

Separate entry and exit for staff and patients will be maintained, with temperature checks and hand sanitisation for all patients entering the building. Patients, essential escorts and staff are to wear face masks or coverings whilst in the building.

The use of telephone triage and consultations were a key feature in aiding recovery planning to reinstate elective clinic schedules for both consultations and treatment clinics under LA, IVS and GA. This process involved contacting all patients who were on a waiting list to be seen for treatment or as new or review patients, and allocating correct categorisation according to Royal College of Surgeons surgical prioritisation list [11]. The classification was from most urgent level 1 to those who could wait more than 3 months to be seen, group 4 . The majority of our cases were deemed to be categorisation 3 and 4 . This also allowed clinicians to take a detailed medical history, clearly identifying those on the shielding list. 


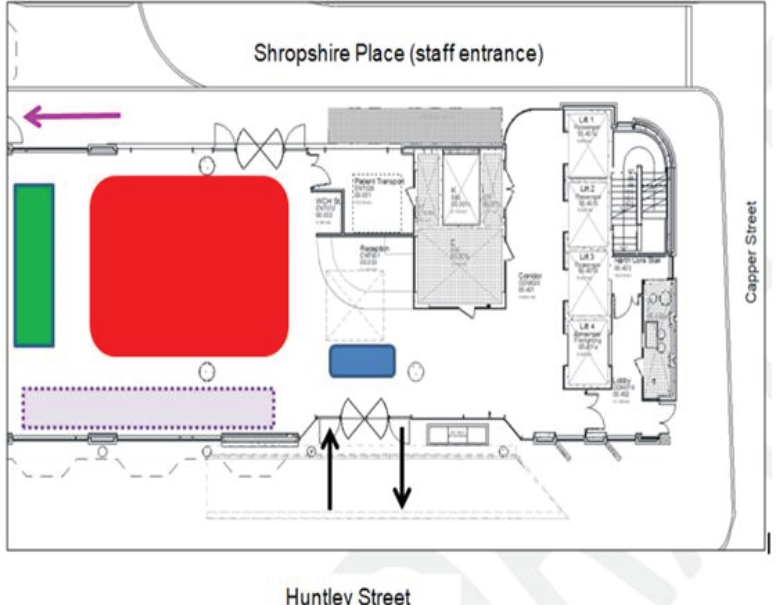

Huntley Street

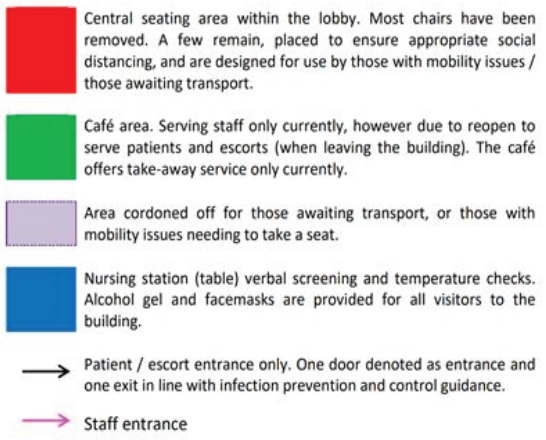

Figure 4: A floor plan of entry and exit routes separating staff and patients.

\section{Discussion}

\section{Personal protective equipment}

The European Standard (EN 149:2001) classifies face filtering respirators into three classes: FFP1, FFP2, and FFP3 with HSE recommended FFP3 respirators offering a minimum filtration efficiency of $99 \%$ [12]. These masks are disposed of after each patient and cost per unit can approximately vary from $£ 1.10$ to $£ 4.80$ [13]. This will have huge cost implications on departments if FFP3 masks are used for all AGPs in the future, with many difficult extractions requiring a surgical handpiece in secondary care settings. In addition, the correct predictability of all extractions being non-surgical or surgical is ambiguous and as Chandler et al. [14] stated both clinical and radiological pre-assessment is required with the only accurate measure being during the procedure itself. It highlights the importance of using careful clinical judgment and treatment planning to don the correct level of PPE and appropriate zone placement for treatment, but also acknowledging all relevant adverse patient factors that will play a role in determining extraction difficulty [15].

During the donning and doffing stages of PPE, the use of a "buddy" or "runner" has been beneficial to the team, in providing assistance and aiding the smooth transition to treatment. This will also have an impact on dedicated roles within a rota and in some cases space allocation for donning and doffing. The HSE has reported that supervised doffing is advantageous, however must not make any physical contact to reduce the risk of exposure [16].

\section{Shielding patients}

As recovery planning stages come to effect, the hospital has opened up clinical time for elective and routine consultations and treatment. This includes many patients who are in the shielding category as listed by NHS Digital. As of $4^{\text {th }}$ June 2020, 3.98\% of England's population are listed in the shielding category [17]. This raises the question of when dentists will be able to provide care for these patients safely and with minimal risk of exposure, with the assumption that this cohort of patients will feel safe to come to a dental hospital setting. Furthermore, it is important as a profession that we are mindful that many shielded patients have not been outside for twelve weeks, and the possibility of now leaving for an elective oral surgical procedure may fill them with anxiety, fear or dread. This could lead to an increase in failure to attend and valuable treatment slots left not utilised, which is a consideration that dentists may need to take into account.

\section{Training and dental education}

Throughout dentistry and medicine, the pandemic has had a significant impact on the current and future profession. Within the dental hospital setting there are many training grades, who have had their training paused and greatly impacted by COVID-19. As recommended by the Chief Dental Officer's guidance, only the necessary staff members should appropriately don PPE and enter the room for an AGP [18]. This will limit the level of teaching available for dental core trainees and specialty trainees, with less one-on-one supervision and through observation of complex cases. This raises the question of the specialty taking a pause in teaching as a whole until true normal activity can resume.

There has been a large upscale to online training including e-learning, webinars and digital conferences. This can prove beneficial for CPD requirements and to aid learning, but it may also fail to provide that personal touch for clinical training, particularly for those in training posts.

\section{Waiting list pressures}

The impact of COVID-19 has created pressure on the waiting lists in the NHS for treatment referred to secondary care. The authors are aware that some patients may have sought urgent treatment at UDCs but a significant number of patients would have postponed their treatment. Considerations should be made regarding the clinical and physical impact extended waiting times may have had on these patients. With the reintroduction of primary care services, it is anticipated that the waiting lists in secondary care will continue to rise.

\section{Treatment options for patients}

A major factor that came to light during the establishment of the service was the treatment of anxious patients for dental extractions, whereby general anaesthetic and intravenous sedation was not an option. During the height of the pandemic, the only option available was local anaesthetic.

Anxious patients have been shown to have delayed presentation for dental care [19] and due to the COVID-19 pandemic had the added stress of coming to a hospital. As clinicians, we identified that this particular cohort of patients had to be taken into special considerations when undertaking dental extractions under local anaesthetic. With the possibility of a second wave, it is important to ensure that appropriate pathways are in place, in the instance of a failed local anaesthetic extraction.

With a significant number of patients, opting for extractions during the lockdown, it is anticipated that this will in turn result in a 
portion of patients seeking gap replacement options. General dental practitioners may need to account for an increased presentation of patients seeking tooth replacement options, following urgent extractions during the pandemic.

\section{Conclusion}

Through the rapid process of setting up an urgent dental care facility in a hospital setting, which was a novel service for the Eastman Dental Hospital, we have learnt considerable lessons which will be valuable in view of a possible second peak or a future pandemic. It has highlighted the need for ready-made protocols and processes in place in the event of a disease outbreak in the future, with allocated roles and responsibilities. The running of the UDC for the first time also shed light upon the challenges of working across departments and demonstrated the need for defined leadership and managerial roles. As we see some normal activity beginning, we are in no doubt likely to achieve close to normal activity again. The question is when, and the effect this momentary pause will have for the future of our profession.

\section{References}

1. Mousavizadeh L, Ghasemi S. Genotype and phenotype of COVID-19: Their roles in pathogenesis. J Microbiol Immunol Infect. 2020.

2. Zhu N, Zhang D, Wang W, Li X, Yang B, Song J, et al. A Novel Coronavirus from Patients with Pneumonia in China, 2019. N Engl J Med. 2020;382(8):727-33.

3. Rapid Evidence Review [Internet]. 2020.

4. Infection Prevention And Control During Health Care When Novel Coronavirus (Ncov) Infection Is Suspected. WHO. 2020.

5. COVID-19 Guidance and Standard Operating Procedure. 2020
6. Guidance: Covid-19 Personal Protective Equipment. 2020.

7. Assessing the evidence base for medical procedures which create a higher risk of respiratory infection transmission from patient to healthcare worker. 2020.

8. Preparedness letter for primary dental care. 2020

9. Bali RK, Chaudhry K. Maxillofacial surgery and COVID-19, The Pandemic!! J Maxillofac Oral Surg. 2020;19(2):159-61.

10. COVID-19: infection prevention and control guidance. 2020.

11. Clinical Guide to Surgical Prioritisation during the Coronavirus Pandemic. Royal College of Surgeons. 2020.

12. Lee SA, Hwang DC, Li HY, Tsai CF, Chen CW, Chen JK. Particle Size-Selective Assessment of Protection of European Standard FFP Respirators and Surgical Masks against Particles-Tested with Human Subjects. J Healthc Eng. 2016;2016: 8572493.

13. Market surveillance of FFP3 disposable respirators. 2020

14. Chandler LP, Laskin DM. Accuracy of radiographs in classification of impacted third molar teeth. J Oral Maxillofac Surg. 1988;46(8):656-60.

15. Renton T, Smeeton N, McGurk M. Factors predictive of difficulty of mandibular third molar surgery. Br Dent J. 2001;190(11):607-10.

16. Evaluation of existing PPE worn by NHS staff for assessment of a patient with a suspected high consequence infectious disease. 2020.

17. Coronavirus shielded patient list open data set E. Coronavirus shielded patient list open data set, England -NHS Digital. 2020

18. Standard Operating Procedure: Transition to Recovery. 2020.

19. Zinke A, Hannig C, Berth H. Comparing oral health in patients with different levels of dental anxiety. Head Face Med. 2018;14(1):25-018-0182-4 